\title{
BIOFILM IMAGE RECONSTRUCTION FOR ASSESSING STRUCTURAL PARAMETERS
}

\author{
Ryan Renslow ${ }^{a}$, Zbigniew Lewandowski ${ }^{b, c}$, and Haluk Beyenal ${ }^{a}{ }^{*}$ \\ a The Gene and Linda Voiland School of Chemical Engineering and Bioengineering, Washington \\ State University, Pullman, WA, USA \\ b Civil and Environmental Engineering, Montana State University, Bozeman, MT, USA \\ c The Center for Biofilm Engineering, Montana State University, Bozeman, MT, USA
}

\begin{abstract}
The structure of biofilms can be numerically quantified from microscopy images using structural parameters. These parameters are used in biofilm image analysis to compare biofilms, to monitor temporal variation in biofilm structure, to quantify the effects of antibiotics on biofilm structure and to determine the effects of environmental conditions on biofilm structure. It is often hypothesized that biofilms with similar structural parameter values will have similar structures; however, this hypothesis has never been tested. The main goal was to test the hypothesis that the commonly used structural parameters can characterize the differences or similarities between biofilm structures. To achieve this goal 1) biofilm image reconstruction was developed as a new tool for assessing structural parameters, 2) independent reconstructions using the same starting structural parameters were tested to see how they differed from each other, 3) the effect of the original image parameter values on reconstruction success was evaluated and 4) the effect of the number and type of the parameters on reconstruction success was evaluated. It was found that two biofilms characterized by identical commonly used structural parameter values may look different, that the number and size of clusters in the original biofilm image affect image reconstruction success and that, in general, a small set of arbitrarily selected parameters may not reveal relevant differences between biofilm structures.
\end{abstract}

\section{Keywords}

biofilm; image; structure; parameters; reconstruction; quantification

\section{INTRODUCTION}

Biofilms affect industrial processes, water and wastewater treatment, environmental ecology and health, and it has been accepted by biofilm researchers that the physical structure of biofilms determines how they interact with their environment. This is because important processes in biofilms such as mass-transport dynamics, hydrodynamics, and microbial community distribution are all related to biofilm structure (deBeer and Stoodley 1995; Picioreanu et al. 2000a; Schramm et al. 1996). It is therefore critically important for researchers to understand and quantify biofilm structure. There has been a significant amount of research effort dedicated to quantifying biofilm structure, and image analysis software packages have been developed to aid in the quantification of biofilm structure

*Corresponding author. beyenal@wsu.edu, Phone: 509-592-7892. 
(Daims et al. 2006; Heydorn et al. 2000; Lewandowski and Beyenal 2007; Milferstedt et al. 2008). Some of the most widely used software include Amira (Visage Imaging, San Diego, California), bioImage_L (de Paz 2009), COMSTAT (Heydorn et al. 2000), DAIME (Daims et al. 2006), ImageJ (National Institutes of Health), Imaris (Bitplane, Zurich, Switzerland), ISA (Beyenal et al. 2004), Matlab with Image Processing Toolbox (The MathWorks, Inc., Natick, Massachusetts), PHLIP (Mueller et al. 2006) and Volocity (PerkinElmer, Waltham, Massachusetts). Since the introduction of the first publicly available packages, the use of structural parameters has become common in a wide array of studies. For example, they have been used to monitor temporal variation in biofilm structure. Jackson et al. (2001) and Heydorn (2000) monitored biofilm structure and showed that structural parameter values were reproducible over short time periods. Also, O'Connell et al. (2007) used structural parameters to track the morphology of Escherichia coli biofilms over time, with and without the presence of antibiotics. In addition to monitoring the temporal development of biofilm structure, there has been much work done on comparing different biofilms using structural parameters. For example, Mah et al. (2003) quantified the structures of a wild type and a mutant strain of Pseudomonas aeruginosa biofilm using total biomass, mean thickness, roughness coefficient, surface area coverage, surface to volume ratio and maximum biofilm thickness and concluded that the differences between the two strains were indiscernible. Recently, Shikuma and Yildiz (2009) tested the effect of selected gene expression on biofilm structure and found critical genes involved in biofilm formation as a response to salinity. They used structural parameters to quantify the changes in biofilm structure. Finally, structural parameters have also been incorporated into biofilm modeling to link biological processes, mass-transport phenomena, and biofilm formation (Picioreanu et al. 1998; Picioreanu et al. 2000a; Picioreanu et al. 2000b).

There are numerous biofilm structural parameters presented in the literature (Daims and Wagner 2007; Milferstedt et al. 2009; Pons et al. 2009; Xavier et al. 2003; Yerly et al. 2008). However, there has been a trend in the literature to use only a limited number of these parameters (de Carvalho and da Fonseca 2007; Luef et al. 2009; Rodriguez and Bishop 2007). As an illustration, Table I shows a list of several peer-reviewed studies since 1990 that used two-dimensional (2D) structural parameters. On average, each study utilized 4 parameters out of the total of 26 different parameters that were used by the studies listed, making cross-comparisons difficult. In part, this trend is justified by the fact that only a handful of the parameters computed from biofilm images, e.g. biofilm porosity (Jackson et al. 2001; Lewandowski et al. 2007; Okabe et al. 1998; Xavier et al. 2005), can be intuitively related to identifiable biological processes, and authors tend to select these parameters in their studies. It is generally believed that the commonly used structural parameters can characterize the differences or similarities between biofilm structures. Selecting the "appropriate" parameters to describe images is a common problem in image analysis, and usually human judgment is used as the ultimate test in selecting these parameters. Furthermore, structural parameters are not well understood because this area of research is still in its infancy. It is often hypothesized that structural parameters are capable of uniquely describing biofilm structure, meaning that two similar looking biofilm images should have nearly identical parameter values and, likewise, two dissimilar images should have different parameter values. This hypothesis has never been tested.

This study explores an approach for testing this hypothesis and for addressing the general problem of selecting meaningful parameters that can describe biofilm structure. If the selected parameters describe biofilms appropriately, then two images having identical parameter values should look similar to each other. It is therefore reasonable to expect that this process can be reversed and that images that look similar to biofilm images can be computer-generated using the structural parameters computed from images of real biofilms. 
The process of generating images using structural parameters is called image reconstruction, and it can be used to generate synthetic images that resemble images of real biofilms.

For image reconstruction to be useful, the reconstructed and the original image must be compared. This requires a definition of similarity. Similar biofilm images will have the same number, size, shape and spatial relation of cell clusters (Rajashekhar and Chaudhuri 2005).

These four basic features uniquely define a binary biofilm image. It is well known in image analysis research that measuring the similarity between two images can be highly subjective (Santini and Jain 1999). In the case of binary biofilm images, the subjectivity comes from difficulty in quantifying two of the four basic features: cell cluster shape and spatial relation. There are literally hundreds of shape and spatial relationship analysis techniques, each used for different purposes. During this study, the difficulty and subjectivity of assessing the similarity between two images was recognized. Traditionally, structural parameters are used to judge whether two biofilm images are quantitatively similar. Because the goal was to determine whether structural parameters themselves can successfully describe biofilm structure, something other than structural parameters had to be used. Therefore it was necessary for a human observer to compare the original and reconstructed images of the biofilm visually. The original and reconstructed images would have identical structural parameters, but it could only be concluded that the structural parameters described the biofilm image uniquely if these images looked similar to the human eye. Therefore, the first step was to calculate a set of structural parameters from a biofilm image. All or a selected set of these parameters were then used for reconstruction, in which a biofilm image that had identical parameter values was generated. If the parameters selected for reconstruction accurately reflected the unique features of the original biofilm structure, then the reconstructed and original biofilm images would look similar. A schematic of this process is given in Figure 1.

The reconstruction process can be used to determine the ability of structural parameters to describe biofilm structure. The main goal was to test the hypothesis that commonly used structural parameters can characterize the differences or similarities between biofilm structures. To achieve this goal 1) biofilm image reconstruction was developed as a new tool for assessing structural parameters, 2) independent reconstructions using the same starting structural parameters were tested to see how they differed from each other, 3) the effect of the original image parameter values on reconstruction success was evaluated and 4) the effect of the number and type of the parameters on reconstruction success was evaluated. Success was measured by how similar the reconstructed image was to the original biofilm image. In this study, 2D light microscopy and single-layer confocal laser scanning microscopy (CLSM) images taken of mixed species biofilms growing under a variety of conditions were used. The following commonly used areal parameters were chosen: areal porosity (AP), average diffusion distance (ADD), maximum diffusion distance (MDD), average horizontal run length (AHRL), average vertical run length (AVRL), fractal dimension (FD) and perimeter (P). Some or all of these parameters were used for each reconstruction process. Even though image quantification is commonly applied to both binary and grayscale 2D and three-dimensional (3D) biofilm images (Beyenal et al. 2004; Daims et al. 2006; Heydorn et al. 2000), 2D binary images were used to introduce the image reconstruction technique. Two-dimensional binary images consider cell clusters as objects and void spaces as background. A grayscale image reconstruction algorithm can be formulated from the methods described in this paper. It is important to note here that grayscale reconstruction would address the type of parameters called textural parameters, as opposed to the areal parameters used in this paper. Textural parameters, such as entropy, energy and homogeneity, have been less popular in quantifying biofilm structure to some extent because their relationship to biofilm processes is less intuitive. This is changing, however, as evidenced by Chinga et al. (2007) and Milferstedt et al. (2008). While future 
work on grayscale and 3D image reconsruction is warranted, many of the findings presented in this manuscript can be generally applied to all types of biofilm image quantification.

\section{MATERIALS AND METHODS}

The methods for growing the biofilms, details of the biofilm reactor used, and details of how the images were acquired are in the Supplementary Material. In this manuscript, a total of 15 different biofilm images that represent a wide range of parameter values are used (Supplementary Material Table S1). For clarity, the binary biofilm images are named "Image A" through "Image O" and are listed in order of decreasing AP (ranging from 0.922 to 0.290 ). All 15 images are presented later in the paper.

\section{Calculating structural parameters}

Areal parameters were calculated for the selected representative biofilm images using the Image Structure Analyzer software package (Beyenal et al. 2004; Lewandowski and Beyenal 2007). Seven commonly used parameters were selected for this analysis: AP, ADD, MDD, AHRL, AVRL, FD and P. The features quantified by these parameters can be related to biofilm processes (Lewandowski and Beyenal 2007). AP is the ratio of the void area to the total image area. The lower the AP, the greater the biofilm surface coverage. ADD is the average distance from each biomass pixel to the nearest void pixel and MDD is the maximum distance from a biomass pixel to the nearest void pixel. Diffusion distances are related to the length that a molecule of the nutrient needs to travel to reach the center of a microcolony. AHRL and AVRL are the average numbers of consecutive biomass pixels horizontally and vertically, respectively. Both are related to the conditions under which the biofilm developed and to the prevailing direction of the shear force in the reactor in which the biofilm accumulated. FD is a measure of biomass perimeter irregularity. The higher the FD, the higher the degree of variability and the roughness of the biofilm cluster surface. It can also be related to the extent to which nutrients are accessible to the surface of a microcolony. $\mathrm{P}$ is the total number of pixels at the biomass boundary, which is also related to the accessibility of the nutrients. These representative structural parameters were chosen because they are commonly used in both the literature and image analysis software packages (Beyenal et al. 2004; Daims et al. 2006; Lewandowski and Beyenal 2007); however, the reconstruction method presented in this article can be used to assess any $2 \mathrm{D}$ areal structural parameter.

\section{Reconstructing biofilm images}

Figure 2 shows the image reconstruction algorithm. One to seven of the calculated parameters are selected and used to reconstruct a new image. At the end of the reconstruction, the new image and the original image have nearly identical values for the selected parameters. The algorithm shown in Figure 2 was integrated into a MATLAB program (64-bit Matlab v7.8 and Image Processing Toolbox, The MathWorks, Inc., Natick, Massachusetts). The program was run on an 8-core, 64-bit Microsoft Windows Vista computer with 16 GB of RAM. The program begins by performing biofilm structure quantification. Reconstruction is performed in the following steps. 1) The software randomly generates an image which has the same AP and dimensions as the original image. Because AP is the only parameter not sensitive to the location of the pixels, it is used as a bootstrapping parameter. To generate a random image with matching AP, biomass pixels are added one at a time to randomly chosen locations on an initially void-covered image. Biomass pixels are added until the AP of the reconstructed image matches that of the original image. 2) The values of the chosen structural parameters are calculated for the randomly generated image. 3) Equation (1), detailed below, is used to calculate the sum of squared differences (SSD) between the structural parameter values of the original image and 
the values calculated for the randomly generated image. 4) Two disparate pixels (one void pixel and one biomass pixel) of the reconstruction image are chosen at random and their locations are switched. 5) The new values of the chosen structural parameters are calculated and 6) the new SSD is then calculated. If SSD has increased, the pixel swap has been unsuccessful and 7a) the pixels are returned to their previous positions. However, if SSD has remained the same or decreased, the pixel swap has been a success and 7b) the pixels remain in their current location. Steps 4-7 are repeated until SSD is less than 0.001 and 8) the reconstruction is complete. This algorithm is based on the fact that all areal parameters other than AP are sensitive to the locations of the pixels. Therefore, forcing the parameters to match is achieved by randomly switching the locations of pixels until the following objective function is minimized:

$$
\mathrm{SSD}=\sum_{\mathrm{i}=1}^{\mathrm{N}}\left(\frac{\mathrm{y}_{\text {reconstructed }, \mathrm{i}}-\mathrm{y}_{\text {original }, \mathrm{i}}}{\mathrm{y}_{\text {original }, \mathrm{i}}}\right)^{2}
$$

Equation 1

In this equation, $\mathrm{N}$ is the total number of parameters used for reconstruction, $\mathrm{i}$ is the arbitrary index value (from 1 to $\mathrm{N}$ ) of the structural parameters (AP, ADD, etc.), $\mathrm{y}_{\text {reconstructed,i }}$ is the structural parameter i value calculated from the reconstructed image and $\mathrm{y}_{\text {original, } \mathrm{i}}$ is the structural parameter i value calculated from the original image. The difference between $\mathrm{y}_{\text {reconstructed, } \mathrm{i}}$ and $\mathrm{y}_{\text {original, } \mathrm{i}}$ is normalized against $\mathrm{y}_{\text {original, }, \mathrm{i}}$, so each selected parameter is weighted equally in the reconstruction. The objective function insures that all parameters are matched concurrently, and not in any determined successive order. During reconstruction, the locations of pixels are continually swapped in the reconstructed image to minimize SSD, while AP remains constant.

Figure 3 shows the progressive steps of three example reconstructions. The steps listed in the figure match the algorithm steps in Figure 2. The second frame shows an image with a random distribution of pixels, but with an AP equal to that of the original image. The reconstructions of Image B, Image I and Image M started with SSD values of 1.9, 4.3 and 4.1 , respectively. Pixels were moved to locations that decreased SSD using algorithm steps 4-7, described in Figure 2. Frames three, four and five show reconstructed images with SSD values that are $25 \%, 50 \%$ and $75 \%$ less than the starting SSD values. For each image in Figure 3, the sixth frame shows the completed reconstruction, with SSD less than 0.001 .

\section{Selecting the optimum SSD value for reconstruction}

Obtaining perfectly matching structural parameters $(\mathrm{SSD}=0)$ was impractical because of the computational time needed to perform the reconstruction. Therefore it was necessary to determine an SSD value that would indicate that the image had been fully reconstructed. To select the optimum SSD value, average accuracy was used as a criterion. The average accuracy, $\mathrm{X}$, is given by:

$$
X=\sum_{i=1}^{N} \frac{x_{i}}{N}
$$

where $\mathrm{x}_{\mathrm{i}}$, the accuracy of structural parameter $\mathrm{i}$ in the reconstructed image, is given by:

$$
\mathrm{x}_{\mathrm{i}}=1-\left|1-\frac{\mathrm{y}_{\text {reconstructed, } \mathrm{i}}}{\mathrm{y}_{\text {original }, \mathrm{i}}}\right|
$$


Average accuracy is related to SSD by the following equation:

$$
\mathrm{SSD}=\mathrm{N}(1-\mathrm{X})^{2}
$$

Equation 4

However, because the AP is always $100 \%$ accurate in the reconstructed images, the equation becomes:

$$
\mathrm{SSD}=\frac{\mathrm{N}^{2}}{\mathrm{~N}-1}(1-\mathrm{X})^{2}
$$

Three biofilm images were reconstructed until the average accuracy surpassed $90 \%, 95 \%$, 97\%, 98\% and 99\%. The resulting reconstructions are shown in Figure 4, as well as the required SSD value for each of the accuracies. Successful pixel swap events seldom result in integer average accuracy values; therefore the images in this figure show the reconstructions when the given average accuracy has just been surpassed. As the average accuracy increases above $95 \%$, the changes seen between images become negligible. From these results, and considering computational time, it was determined that if SSD is smaller than 0.001 the reconstructed and original images have comparable structural parameters and reconstruction is complete. An SSD of 0.001 for seven parameters means that the average accuracy is $98.9 \%$. The time it took for a reconstruction to reach a $98.9 \%$ average accuracy was generally several hours to a day less than that needed to reach $99 \%$.

\section{Original and reconstructed image comparison}

There is currently no quantitative method for accurately comparing two biofilm images. This study relied on the human visual system (HVS). The HVS is capable of qualitatively comparing images, and is often more successful than computer-based methods in image analysis tasks (Loncaric 1998; Marr 1985; Samal and Iyengar 1992). To measure the success of the structural parameters in 15 image reconstructions, 60 volunteers were asked to rank the pairs of images from 1 to 15,1 being "most similar" and 15 being "least similar." The volunteers included biofilm researchers, other scientists and nonscientists. While comparison using the HVS is open to biases, the results obtained in this study have important implications for using biofilm image reconstruction and for using biofilm structural parameters in general. Image subtraction was also tested to evaluate success of reconstruction (see Supplementary Materials). Based on a Pearson product-moment correlation coefficient (PMCC) of -0.27 (Supplementary Materials Figure S2), we concluded that there was no significant correlation between the HVS survey results and the subtraction score. Possible reasons for the poor correlation are exemplified with Supplementary Materials Figure S3.

\section{RESULTS AND DISCUSSION}

\section{Reconstruction of single images multiple times}

Figure 5 shows six original biofilm images and the results of three independent reconstructions of each one using structural parameters AP, ADD, MDD, AHRL, AVRL, FD and $\mathrm{P}$. Each of the independent reconstructions started with a different random distribution of pixels and had different random pixel swaps during the reconstruction process. Although it is possible to see similarities between the original and reconstructed images, the extent of the similarity is not satisfactory. To a human observer they do not look identical. From this it can be concluded that even using all seven parameters is not enough to reconstruct identical-looking biofilm images. It is possible to have two biofilms with 
identical structural parameters that look physically different. Note that while the original and the reconstructed images are visually different, the independently reconstructed images look more similar to each other.

\section{Success of reconstruction}

Figure 6 shows all 15 biofilm images used in this study and the corresponding reconstructions. Again, the original and reconstructed images do not look identical, but some show more similarity than others. Figure 7 shows the results of the survey in which volunteers were asked to rank the similarity of the pairs shown in Figure 6. The most similar image pairs are for Images I, D, J, G and L. There was a high consensus on these five pairs, and their standard deviations were relatively low (average of 2.4). The next nine pairs had higher standard deviations (average of 3.3) and very similar ranks. The success of these nine pairs was hard for the volunteers to differentiate. The least successful pair, Image K, was nearly two ranks away from the next worst pair, clearly identifying it as being the "least similar." To test the hypothesis that the starting image parameter values affect the success of the reconstruction process, the Pearson product-moment correlation coefficient was calculated between each structural parameter and the success ranking of each reconstructed image. PMCCs range from values of -1 to 1 and measure correlation: a larger magnitude represents a stronger correlation and zero represents no correlation. Positive and negative PMCCs denote proportionality and inverse proportionality, respectively. No strong correlations were found. The highest correlations were with ADD and MDD, which had PMCCs of 0.44 and 0.42 , respectively. Both of these parameters give information about the size of the cell clusters. Table II shows the ADD, the MDD, the average largest cluster size and the average number of clusters per image for the five most, five middle and five least similar image pairs as ranked by the survey. The five most similar reconstructions had more cell clusters and smaller maximum cluster sizes than the five least similar reconstructions. It is hypothesized that this is due to the nature of the HVS: it is easier for human eyes to see differences between large cell clusters because the details of cluster shape and orientation are much more visible than those of small cell clusters. Survey participants could immediately spot many differences when the biggest cluster size was very large; when it was smaller, details were not as apparent. While there are no statistically strong correlations between success of reconstruction and starting image parameter values, biofilm images with numerous small cell clusters resulted in more similar reconstructions than ones with sparse large cell clusters. It is hypothesized that biofilm images with only a few large cell clusters may be less uniquely defined by the seven structural parameters than biofilm images with numerous small clusters. The size of the cell clusters appears to be important for structure comparison studies because reconstruction success and structural parameters are dependent on it. Future studies should focus on developing quantitative methods for comparing original and reconstructed images. A nonsubjective and quantitative method is needed in order to test this hypothesis and advance the uses of the reconstruction technique.

\section{Changing the number and order of parameters}

How the number and order of parameters would affect reconstruction was also tested. Figure 8 shows the results of reconstruction using progressively more parameters. Three different tracks were explored, shown in Table III. These three tracks were chosen out of the 720 different possible tracks based on previous experience using biofilm structural parameters. Three tracks were selected that were expected to provide the biggest differences in reconstruction.

When only AP was used, the reconstructed images showed no similarity to their original images. When two structural parameters were used (AP+P, AP+ADD, or AP+AHRL), the reconstructed images were significantly different from the original images. However, for 
track 1 and track 3 , after only three parameters had been used, the results were moderately comparable to those obtained using all seven parameters. For track 2 , it took six parameters before the results became comparable. It can be concluded that for the selected image there is overlap in the information that the parameters provide about the biofilm structure. For the chosen 15 images, ADD, MDD, AHRL and AVRL were found to be correlated to each other with an average PMCC of 0.88 . Notice that in all three tracks, small few-pixel artifacts are added to the reconstructed images with the addition of FD. This appears to be the main reason that track 2 took six parameters before becoming comparable, and this could explain why track 3 became comparable faster despite highly correlated parameters being used. The addition of FD slows down reconstruction unless it is used in combination with several other parameters. It can be concluded that certain combinations of parameters are better than others, and that in general more parameters are better than fewer for uniquely describing biofilm structure. However, note that the parameters selected for biofilm structure studies should not correlate; otherwise the parameters will produce redundant information.

\section{Implications and future outlook}

As demonstrated by the image pairs in Figure 6, it is possible for significant changes to occur in biofilm structure that would go undetected by the commonly used structural parameters. For example, in a hypothetical study on the effect of a chemical that blocks microbial quorum sensing on biofilm structure, the results might appear identical to Image $\mathrm{H}$ in Figure 6. Consider that the original image represents the biofilm formation without the chemical, while the reconstructed image represents the biofilm formation with the chemical. Researchers could easily identify differences between the two biofilm structures and claim that the chemical had changed a biological process in the biofilm. They might notice the decreased coordination in cluster orientation, the greater dispersion of clusters in the biofilm space, the increase in round cluster shapes, or the greater number of exceptionally small clusters. However, when they calculated the commonly used structural parameters to quantify these changes, the parameter values would not reveal any difference between the images because the parameter values of the two images would be identical. Image $\mathrm{H}$ gives a blatant and possibly exaggerated example of the shortcomings of the current, commonly used 2D structural parameters. It is easy to imagine that less obvious differences might not be detected. A set of parameters that could uniquely define biofilm structure would reveal much finer detail than is currently possible.

Finding such a set of parameters is possible if researchers use image reconstruction as a tool for selecting better structural parameter sets, the ultimate goal being to determine the smallest set of parameters that can uniquely define a biofilm. The next step in finding this small uniquely defining set will be a much bigger challenge than showing that the current commonly used structural parameters are insufficient, especially since computer-based image comparison is almost certainly a prerequisite. Once an objective computer-based method is developed to determine reconstruction success, a rigorous test of structural parameters, both new and old, can be performed to determine which parameter sets best define biofilm structure. Researchers should start with a small set size and test all combinations of parameters of this set size. After this, the set size may be increased incrementally while all combinations are tested until a sufficient set is found. Possible new parameters include: number of clusters, average cluster surface area, maximum cluster surface area and average cluster angle. Many more parameters could be designed to help characterize biofilm structure, especially to address the subject of cluster spatial relation, which is an area that currently lacks sufficient parameters. Efficient algorithms may be developed to bypass the brute force method by honing in on the parameters that consistently produce good reconstructions, and the use of parallel computing should be used to decrease computation time for larger, grayscale, or 3D image reconstructions. Furthermore, a decision 
will have to be made about how much structural information should be defined in a parameter set. A sufficiently large set could characterize biofilm structure entirely, down to single-pixel resolution, but would be too big for practical use and would lose the ability to highlight the biologically significant features. The goal will be to minimize the parameter set size while maximizing its characterizing power. Finally, the best set of parameters for quantifying biofilm structure appears to be dependent on the scale of the images used because of the dependence of reconstruction success on cluster size. This is well supported by other reports on the effect of representative elemental area on biofilm structure quantification (Milferstedt et al. 2009). Recently, Wagner et al. (2010) used structural parameters to investigate the mesoscale structure of biofilms that had been imaged using optical coherence tomography. They compared the mesoscale structural parameters to microscale structural parameters from CLSM images and found that there were some significant differences. Thus, image reconstruction should be used to determine what role image scale has on the choice of parameters to be used in biofilm structure comparison studies.

\section{CONCLUSIONS}

An algorithm for reconstructing biofilm images using structural parameters was developed and implemented. Image reconstruction can be used as a tool for determining whether the parameters selected to characterize biofilm structure are unique descriptors of biofilm structure. In the future, new parameters should be tested using the presented algorithm to determine whether they aid in describing biofilm structure uniquely. This study concludes that 1) two biofilms characterized by identical commonly used structural parameter values may look different, 2) the number and size of clusters in the original biofilm image affect image reconstruction success, and 3) in general, a small set of arbitrarily selected parameters may not reveal relevant differences between biofilm structures.

\section{Supplementary Material}

Refer to Web version on PubMed Central for supplementary material.

\section{Acknowledgments}

This research was supported by a 3M Nontenured Faculty Grant. NIH Training Grant T32-GM008336 helped fund Ryan Renslow during this project.

\section{References}

Battin TJ, Kaplan LA, Newbold JD, Cheng XH, Hansen C. Effects of current velocity on the nascent architecture of stream microbial biofilms. Applied and Environmental Microbiology. 2003; 69(9): 5443-5452. [PubMed: 12957933]

Beyenal H, Lewandowski Z. Mass-transport dynamics, activity, and structure of sulfate-reducing biofilms. Aiche Journal. 2001; 47(7):1689-1697.

Beyenal H, Lewandowski Z, Harkin G. Quantifying biofilm structure: Facts and fiction. Biofouling. 2004; 20(1):1-23. [PubMed: 15079889]

Chinga G, Johnsen PO, Dougherty R, Berli EL, Walter J. Quantification of the 3D microstructure of SC surfaces. Journal of Microscopy-Oxford. 2007; 227(3):254-265.

Daims H, Lucker S, Wagner M. daime, a novel image analysis program for microbial ecology and biofilm research. Environmental Microbiology. 2006; 8(2):200-213. [PubMed: 16423009]

Daims H, Wagner M. Quantification of uncultured microorganisms by fluorescence microscopy and digital image analysis. Applied Microbiology and Biotechnology. 2007; 75(2):237-248. [PubMed: 17333172] 
de Carvalho C, da Fonseca MMR. Assessment of three-dimensional biofilm structure using an optical microscope. Biotechniques. 2007; 42(5):616-620. [PubMed: 17515200]

de Paz LEC. Image Analysis Software Based on Color Segmentation for Characterization of Viability and Physiological Activity of Biofilms. Applied and Environmental Microbiology. 2009; 75(6): 1734-1739. [PubMed: 19139239]

deBeer D, Stoodley P. Relation between the structure of an aerobic biofilm and transport phenomena? Water Science and Technology. 1995; 32(8):11-18.

Heydorn A, Nielsen AT, Hentzer M, Sternberg C, Givskov M, Ersboll BK, Molin S. Quantification of biofilm structures by the novel computer program COMSTAT. Microbiology-Uk. 2000; 146:2395-2407.

Jackson G, Beyenal H, Rees WM, Lewandowski Z. Growing reproducible biofilms with respect to structure and viable cell counts. Journal of Microbiological Methods. 2001; 47(1):1-10. [PubMed: 11566221]

Kim HY, Yeon KM, Lee CH, Lee S, Swaminathan T. Biofilm structure and extracellular polymeric substances in low and high dissolved oxygen membrane bioreactors. Separation Science and Technology. 2006; 41(7):1213-1230.

Kim JW, Choi H, Pachepsky YA. Biofilm morphology as related to the porous media clogging. Water Research. 2010; 44(4):1193-1201. [PubMed: 19604533]

Lewandowski, Z.; Beyenal, H. Fundamentals of Biofilm Research. Boca Raton, FL: CRC Press Taylor \& Frances Group; 2007.

Lewandowski Z, Beyenal H, Myers J, Stookey D. The effect of detachment on biofilm structure and activity: the oscillating pattern of biofilm accumulation. Water Science and Technology. 2007; 55(8-9):429-436. [PubMed: 17547014]

Lewandowski Z, Beyenal H, Stookey D. Reproducibility of biofilm processes and the meaning of steady state in biofilm reactors. Water Science and Technology. 2004; 49(11-12):359-364. [PubMed: 15303762]

Lewandowski Z, Webb D, Hamilton M, Harkin G. Quantifying biofilm structure. Water Science and Technology. 1999; 39(7):71-76.

Lomander A, Schreuders P, Russek-Cohen E, Ali L. A method for rapid analysis of biofilm morphology and coverage on glass and polished and brushed stainless steel. Transactions of the Asae. 2002; 45(2):479-487.

Loncaric S. A survey of shape analysis techniques. Pattern Recognition. 1998; 31(8):983-1001.

Luef B, Neu TR, Zweimuller I, Peduzzi P. Structure and Composition of Aggregates in Two Large European Rivers, Based on Confocal Laser Scanning Microscopy and Image and Statistical Analyses. Applied and Environmental Microbiology. 2009; 75(18):5952-5962. [PubMed: 19633114]

Mah TF, Pitts B, Pellock B, Walker GC, Stewart PS, O'Toole GA. A genetic basis for Pseudomonas aeruginosa biofilm antibiotic resistance. Nature. 2003; 426(6964):306-310. [PubMed: 14628055]

Marr, D. Vision. W.H. Freeman \& Co Ltd; 1985.

Milferstedt K, Pons MN, Morgenroth E. Textural fingerprints: A comprehensive descriptor for biofilm structure development. Biotechnology and Bioengineering. 2008; 100(5):889-901. [PubMed: 18551529]

Milferstedt K, Pos MN, Morgenroth E. Analyzing Characteristic Length Scales in Biofilm Structures. Biotechnology and Bioengineering. 2009; 102(2):368-379. [PubMed: 18949757]

Mueller LN, de Brouwer JFC, Almeida JS, Stal LJ, Xavier JB. Analysis of a marine phototrophic biofilm by confocal laser scanning microscopy using the new image quantification software PHLIP. BMC Ecology. 2006:6. [PubMed: 16681850]

O'Connell HA, Niu C, Gilbert ES. Enhanced high copy number plasmid maintenance and heterologous protein production in an Escherichia coli biofilm. Biotechnology and Bioengineering. 2007; 97(3):439-446. [PubMed: 17058286]

Obert M, Pfeifer P, Sernetz M. Microbial-growth patterns described by fractal geometry. Journal of Bacteriology. 1990; 172(3):1180-1185. [PubMed: 2106504]

Okabe S, Kuroda H, Watanabe Y. Significance of biofilm structure on transport of inert particulates into biofilms. Water Science and Technology. 1998; 38(8-9):163-170. 
Picioreanu C, van Loosdrecht MCM, Heijnen JJ. Mathematical modeling of biofilm structure with a hybrid differential-discrete cellular automaton approach. Biotechnology and Bioengineering. 1998; 58(1):101-116. [PubMed: 10099266]

Picioreanu C, van Loosdrecht MCM, Heijnen JJ. Effect of diffusive and convective substrate transport on biofilm structure formation: A two-dimensional modeling study. Biotechnology and Bioengineering. 2000a; 69(5):504-515. [PubMed: 10898860]

Picioreanu C, van Loosdrecht MCM, Heijnen JJ. A theoretical study on the effect of surface roughness on mass transport and transformation in biofilms. Biotechnology and Bioengineering. 2000b; 68(4):355-369. [PubMed: 10745204]

Pons MN, Milferstedt K, Morgenroth E. Biofilm Monitoring on Rotating Discs by Image Analysis. Biotechnology and Bioengineering. 2009; 103(1):105-116. [PubMed: 19132742]

Purevdorj B, Costerton JW, Stoodley P. Influence of hydrodynamics and cell signaling on the structure and behavior of Pseudomonas aeruginosa biofilms. Applied and Environmental Microbiology. 2002; 68(9):4457-4464. [PubMed: 12200300]

Raajan R, Veluchamy A, Beyenal H, Lewandowski Z. Characterizing temporal development of biofilm porosity using artificial neural networks. Water Science and Technology. 2008; 57(12): 1867-1872. [PubMed: 18587172]

Rajashekhar, Chaudhuri S. Logo retrieval using morphological pattern spectrum. Iete Journal of Research. 2005; 51(5):339-351.

Rodriguez SJ, Bishop PL. Three-dimensional quantification of soil biofilms using image analysis. Environmental Engineering Science. 2007; 24(1):96-103.

Samal A, Iyengar PA. Automatic recognition and analysis of human faces and facial expressions - a survey. Pattern Recognition. 1992; 25(1):65-77.

Santini S, Jain R. Similarity measures. Ieee Transactions on Pattern Analysis and Machine Intelligence. 1999; 21(9):871-883.

Schramm A, Larsen LH, Revsbech NP, Ramsing NB, Amann R, Schleifer KH. Structure and function of a nitrifying biofilm as determined by in situ hybridization and the use of microelectrodes. Applied and Environmental Microbiology. 1996; 62(12):4641-4647. [PubMed: 8953735]

Shikuma NJ, Yildiz FH. Identification and Characterization of OscR, a Transcriptional Regulator Involved in Osmolarity Adaptation in Vibrio cholerae. Journal of Bacteriology. 2009; 191(13): 4082-4096. [PubMed: 19329635]

Wagner M, Taherzadeh D, Haisch C, Horn H. Investigation of the mesoscale structure and volumetric features of biofilms using optical coherence tomography. Biotechnology and Bioengineering. 2010; 107(5):844-853. [PubMed: 20717977]

Xavier JB, White DC, Almeida JS. Automated biofilm morphology quantification from confocal laser scanning microscopy imaging. Water Science and Technology. 2003; 47(5):31-37. [PubMed: 12701903]

Xavier, JdB; Picioreanu, C.; van Loosdrecht, MCM. A general description of detachment for multidimensional modelling of biofilms. Biotechnology and Bioengineering. 2005; 91(6):651669. [PubMed: 15918167]

Yang XM, Beyenal H, Harkin G, Lewandowski Z. Quantifying biofilm structure using image analysis. Journal of Microbiological Methods. 2000; 39(2):109-119. [PubMed: 10576700]

Yerly J, Hu YP, Martinuzzi RJ. Biofilm structure differentiation based on multi-resolution analysis. Biofouling. 2008; 24(5):323-337. [PubMed: 18568669]

Zahid WM, Ganczarczyk JJ. Fractal properties of the RBC biofilm structure. Water Science and Technology. 1994a; 29(10-11):271-279.

Zahid WM, Ganczarczyk JJ. Structure of RBC biofilms. Water Environment Research. 1994b; 66(2): $100-106$. 

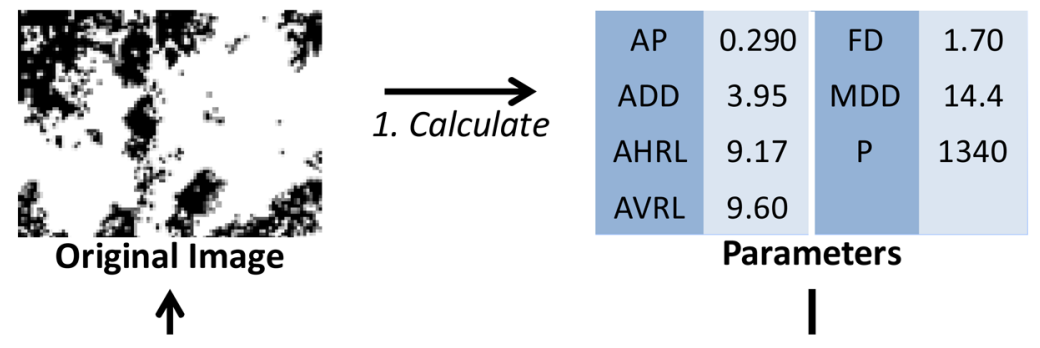

Parameters
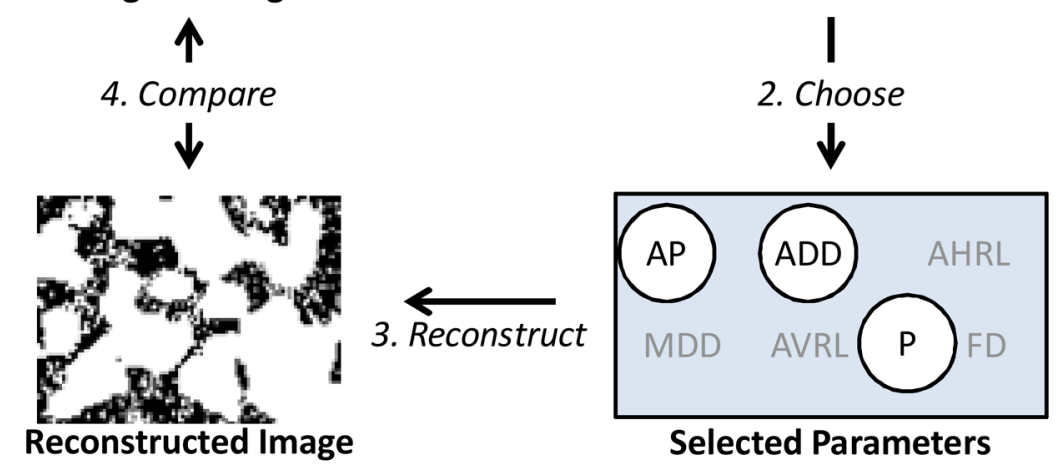

Selected Parameters

Figure 1.

Schematic description of how image reconstruction is used as a tool for assessing structural parameters and their ability to uniquely describe biofilm structure. 1) First, biofilm structure quantification is used to determine structural parameter values for the original image. 2) Some combination of the calculated parameters is chosen for constructing a new image. The number of parameters chosen can range from one to several. 3) The selected parameters are then used to construct a new image that has identical structural parameter values. 4) Finally, the reconstructed image is compared to the original image. If the reconstructed image is similar to the original image, then the selected parameters uniquely describe the biofilm structure. 


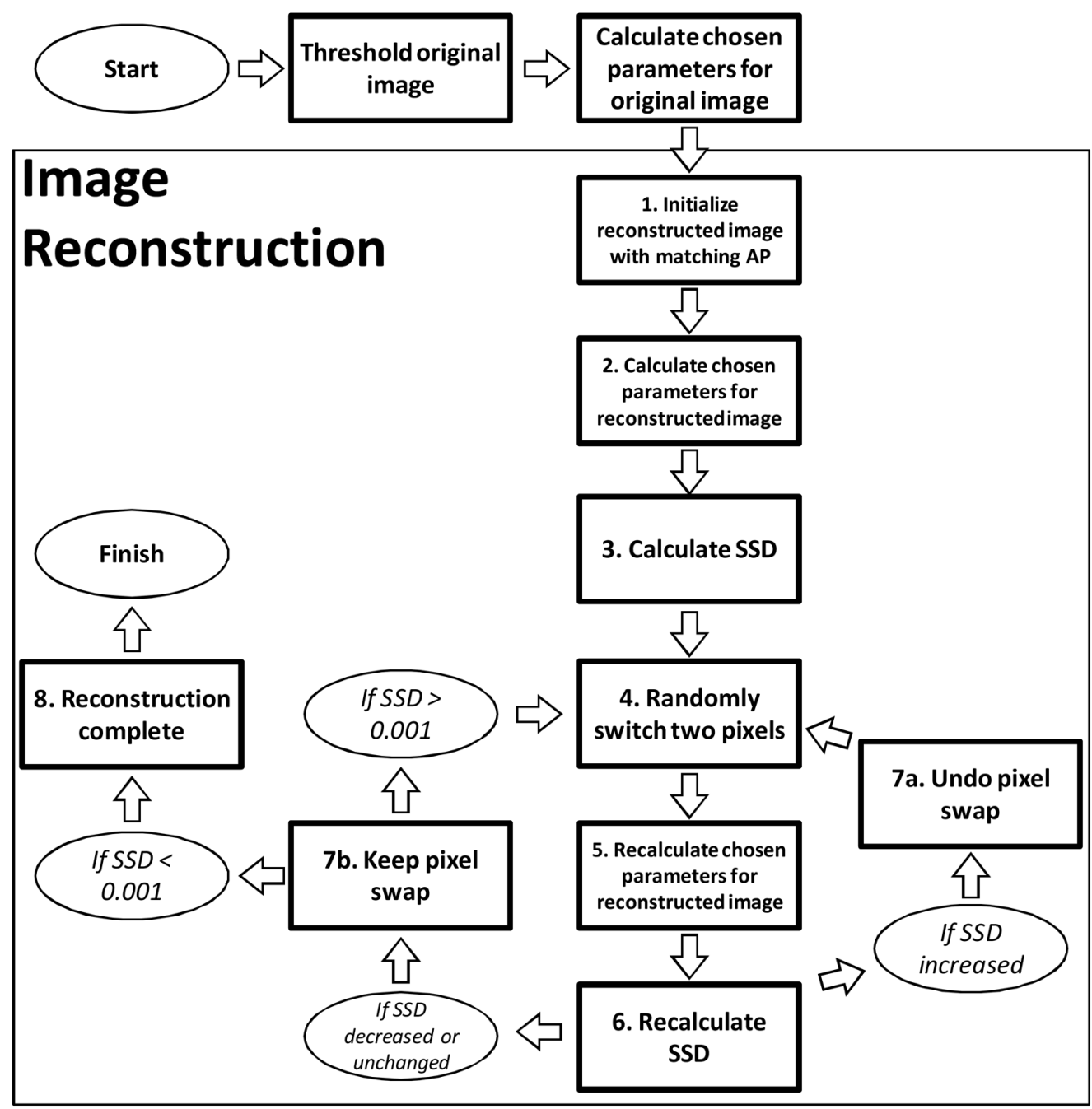

Figure 2.

Algorithm for reconstructing biofilm images using a selected set of image parameters. Biofilm structure quantification steps are shown outside the large box, and image reconstruction steps are shown inside the large box, starting at step 1. 


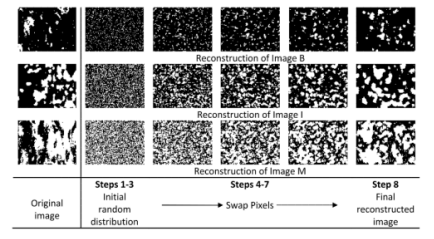

Figure 3.

Progression of reconstruction for three biofilm images, matching seven structural parameters. After the initial random distribution, each frame shows an equal and consecutive reduction in SSD. The SSD of the final reconstructed image is less than 0.001 . 


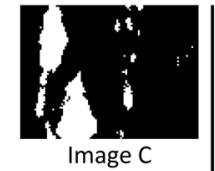

AP $\quad 0.841$

ADD $\quad 3.07$

MDD $\quad 9.90$

AHRL $\quad 6.62$

AVRL $\quad 10.2$

FD $\quad 1.17$

P $\quad 3600$

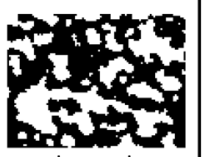

Image $L$

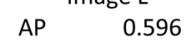

ADD $\quad 2.08$

MDD $\quad 8.25$

AHRL $\quad 7.56$

AVRL $\quad 6.06$

FD $\quad 1.85$

$\begin{array}{ll}P & 1170\end{array}$

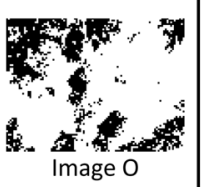

Image $O$

AP $\quad 0.290$

ADD $\quad 3.95$

MDD $\quad 14.4$

AHRL $\quad 9.17$

AVRL $\quad 9.60$

FD $\quad 1.70$

P $\quad 1340$

Original image

Average accuracy Average accuracy Average accuracy Average accuracy Average accuracy

$\mathrm{SSD} \leq 8.17 \mathrm{e}-2$

$\mathrm{SSD} \leq 2.04 \mathrm{e}-2$

$\mathrm{SSD} \leq 7.35-3$

$\mathrm{SSD} \leq 3.27 \mathrm{e}-3$

$\mathrm{SSD} \leq 8.17 \mathrm{e}-4$

Figure 4.

Reconstruction of three images to five levels of average structural parameter accuracy and the corresponding SSD values. Structural parameter values are listed for each original image and its reconstructed images. 


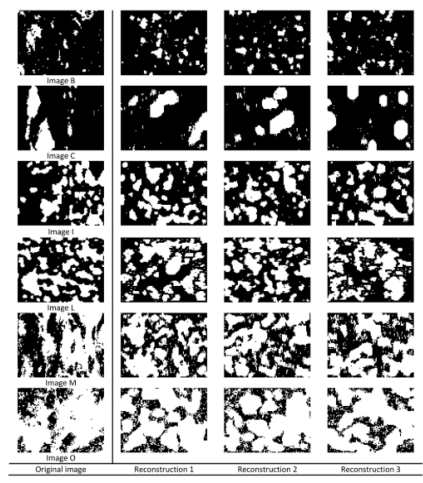

Figure 5.

Three independent reconstructions of six different biofilm images matching AP, ADD, MDD, AHRL, AVRL, FD and P. Each of the independent reconstructions started with a different random distribution of pixels and had different random pixel swaps during the reconstruction process. 


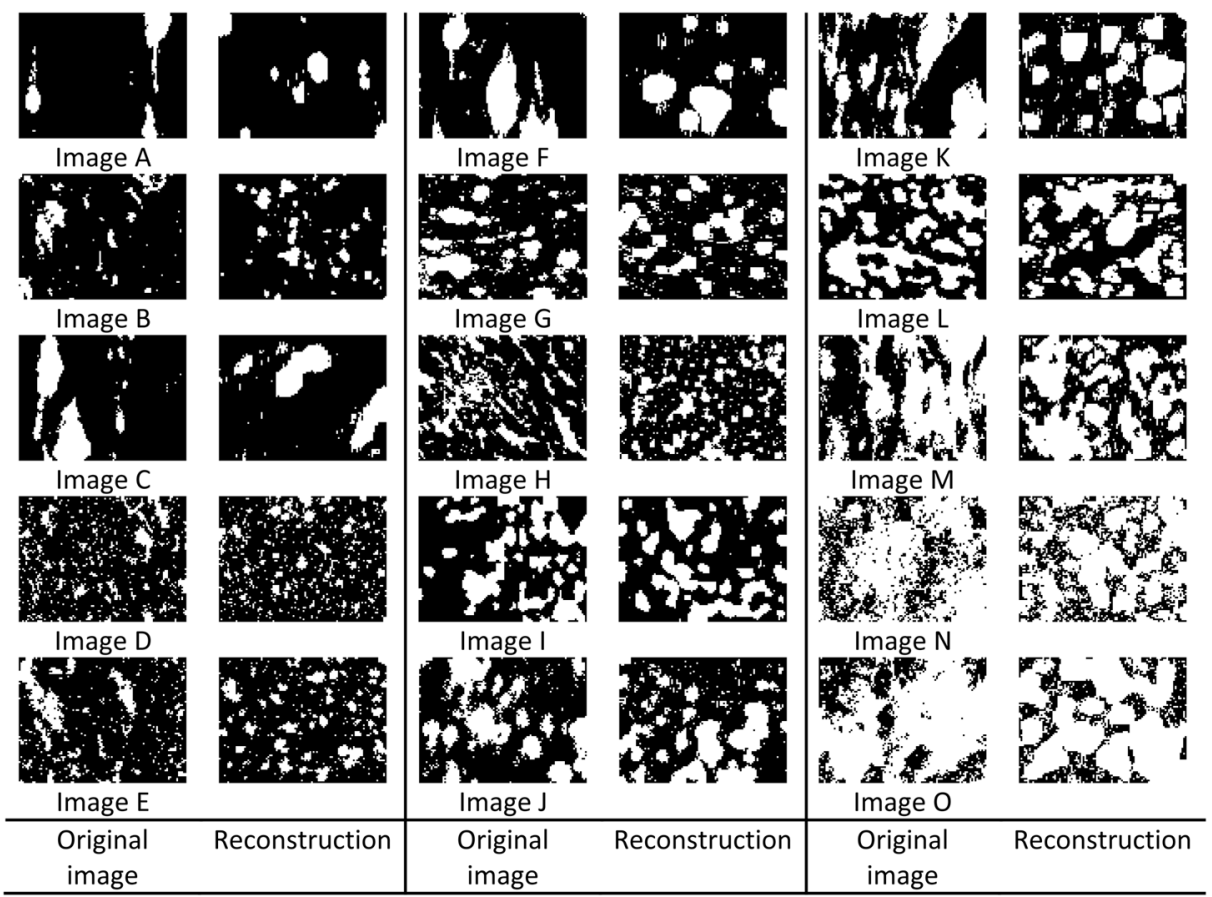

Figure 6.

Fifteen biofilm images and corresponding reconstructions matching AP, ADD, MDD, AHRL, AVRL, FD and P. 


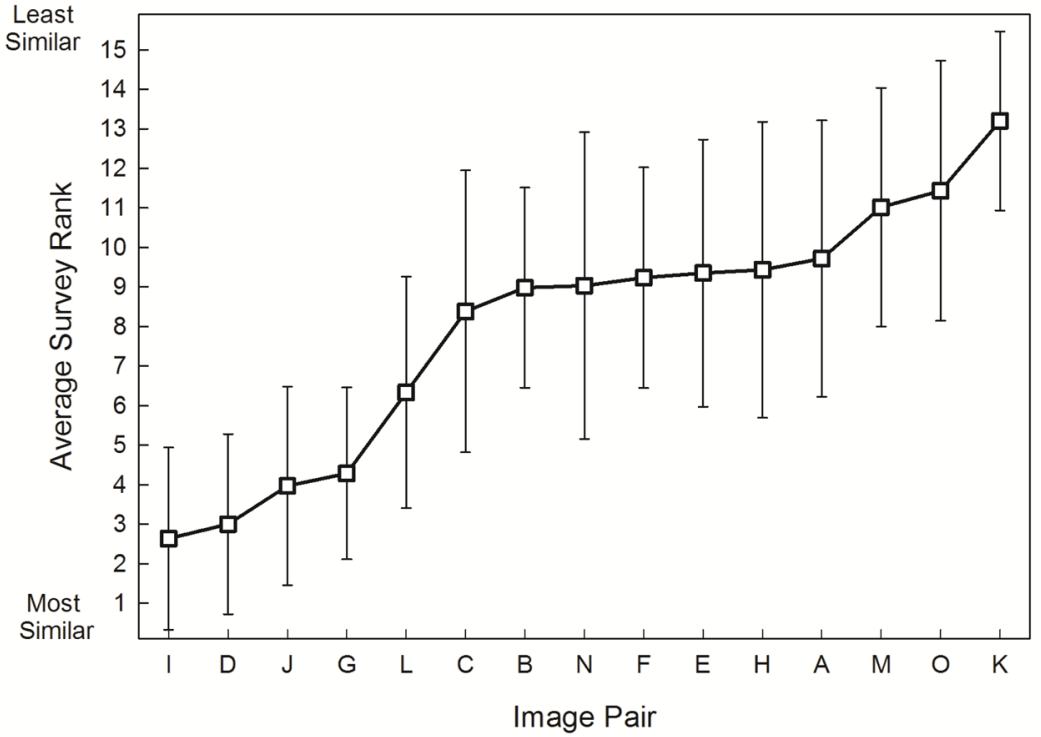

Figure 7.

Results of the survey asking 60 volunteers to rank the 15 pairs of biofilm images and their corresponding reconstructed images from 1 to 15 based on similarity, 1 being "most similar" and 15 being "least similar." Single standard deviation error bars are shown. 


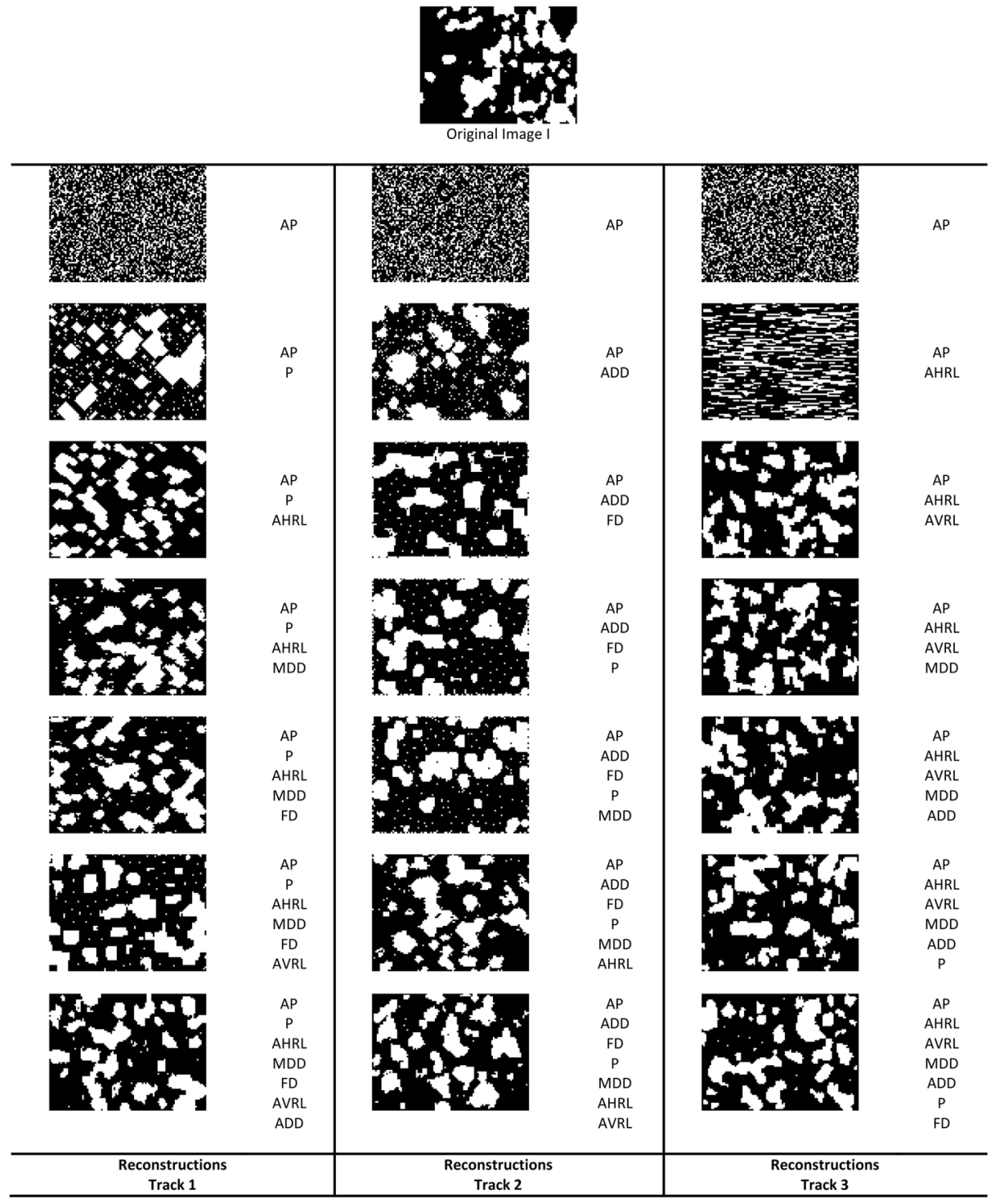

Figure 8.

Image reconstruction using different sets of selected parameters. Each track starts with one parameter and adds one at a time until all seven parameters have been used. 


\section{Table I}

List of several studies that used 2D structural parameters. The parameters used include angular second moment (ASM), areal porosity (AP), average diffusion distance (ADD), average horizontal run length (AHRL), average vertical run length (AVRL), biofilm coverage (BC), biofilm diameter (BD), biofilm fragmentation $(\mathrm{BF})$, biofilm patch area (BPA), biofilm patch circularity (BPC), biofilm patch perimeter (BPP), energy (E), fractal dimension (FD), homogeneity $(\mathrm{H})$, inverse difference moment (IDM), lacunarity (L), maximum diffusion distance (MDD), maximum pore dimension (MPD), mean diameter of macropores (MDM), perimeter (P), pore size (PS), specific biofilm contacts (SBC), specific biofilms (SB), specific surface area (SSA), surface area (SA) and textual entropy (TE).

\begin{tabular}{|l|l|l|}
\hline Reference & Microorganism & 2D Parameters \\
\hline Kim et al. 2010 & Pseudomonas putida & E, FD, H, L, SBC, SB, TE \\
\hline Raajan et al. 2008 & KP\&P* & AP, ADD, AHRL, AVRL, FD, TE \\
\hline Kim et al. 2006 & mixed species & AP \\
\hline Lewandowski et al. 2004 & KP\&P & AP \\
\hline Beyenal et al. 2004 & KP\&P & AP, ADD, AHRL, AVRL, E, FD, H, MDD, P, TE \\
\hline Battin et al. 2003 & mixed species & AP, BF \\
\hline Purevdorj et al. 2002 & Pseudomonas aeruginosa & ASM, AP, ADD, AHRL, AVRL, FD, IDM, MDD \\
\hline Lomander et al. 2002 & Escherichia coli & BC, BPA, BPC, BPP \\
\hline Jackson et al. 2001 & KP\&P & AP, ADD, FD, TE \\
\hline Beyenal and Lewandowski 2001 & $\begin{array}{l}\text { Desulfovibrio desulfuricans Pseudomonas } \\
\text { fluorescens }\end{array}$ & AP, FD \\
\hline Yang et al. 2000 & KP\&P & ASM, AR, ADD, AHRL, AVRL, FD, IDM, MDD, TE \\
\hline Lewandowski et al. 1999 & KP\&P & AP, FD, MDD, TE \\
\hline Okabe et al. 1998 & mixed species & AP, MDM, SSA \\
\hline Zahid and Ganczarczyk 1994a & mixed species & AP, FD, MPD, P, PS, SA \\
\hline Zahid and Ganczarczyk 1994b & mixed species & AP, MPD \\
\hline Obert et al. 1990 & Ashbya gossypii Streptomyces griseus & BD, FD \\
\hline
\end{tabular}

KP\&P = Klebsiella pneumoniae, Pseudomonas aeruginosa and Pseudomonas fluorescens 


\section{Table II}

Comparison of number of clusters and largest cluster size for three categories of reconstructed images as ranked by HVS.

\begin{tabular}{lcccc}
\hline Pairs & Average ADD & Average MDD & Average largest cluster size & Average \# of clusters \\
\hline $\mathbf{5}$ most similar & 1.85 & 6.95 & 616 & 132 \\
$\mathbf{5}$ middle & 2.22 & 7.57 & 1226 & 97 \\
$\mathbf{5}$ least similar & 2.54 & 9.50 & 1817 & 87 \\
\hline
\end{tabular}




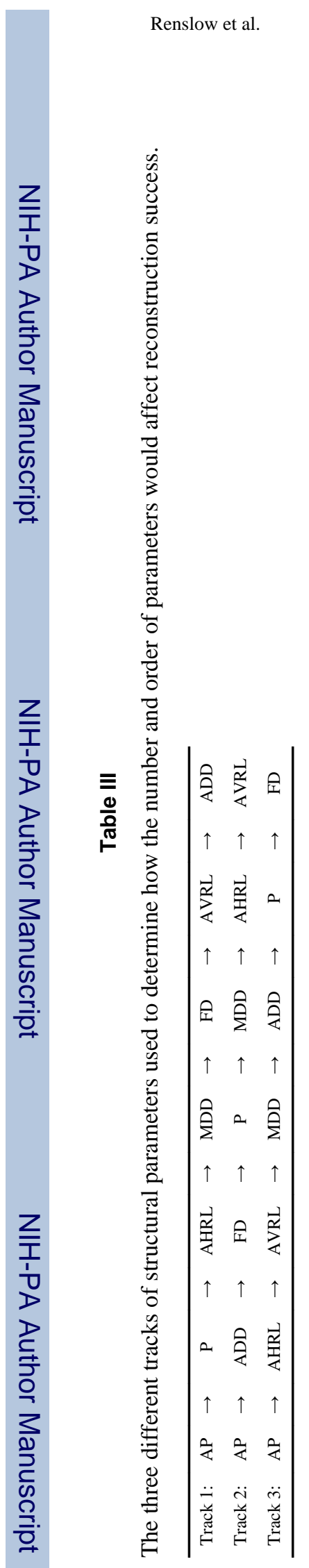

Page 22

Biotechnol Bioeng. Author manuscript; available in PMC 2012 June 1. 